\title{
What to do when perioperative point-of-care ultrasound shows evidence of a full stomach despite fasting?
}

\author{
Stephan K. W. Schwarz, MD, PhD, FRCPC (iD - Christopher Prabhakar, MD, FRCPC
}

Received: 2 March 2020 / Accepted: 2 March 2020/Published online: 1 May 2020

(C) Canadian Anesthesiologists' Society 2020

The tenth Law of The House of God-the cult 1978 novel about medical internship_-states, "If you don't take a temperature, you can't find a fever." As anesthesiologists, we have historically not been at the forefront of this law's decidedly cynical tenet-namely to avoid actively searching for problems. Quite the opposite: anesthesiology in particular has been a specialty that throughout its history has seen the advent and introduction of an array of innovative "thermometers," i.e., monitoring tools that aid real-time detection of untoward pathophysiologic states to make them amenable for rapid clinical decision-making. Arguably, the most notable examples include measuring blood oxygenation by oxygen electrodes and later by pulse oximetry, ${ }^{2,3}$ capnometry, ${ }^{4}$ and anesthetic agent monitoring. ${ }^{5}$

Nevertheless, it takes time for such diagnostic tools to become ubiquitous clinical standards. For example, the 2012 revised edition of the Canadian Anesthesiologists' Society Guidelines to the Practice of Anesthesia recommended that capnography monitoring be utilized in all patients undergoing general anesthesia and deeper levels of sedation ${ }^{6}$ (this was expanded to a consideration for all levels of sedation in 2019). ${ }^{7}$ Speaking of thermometers, ${ }^{8}$ the critical importance of maintaining patient normothermia perioperatively was emphasized in

S. K. W. Schwarz, MD, PhD, FRCPC ( $)$.

C. Prabhakar, MD, FRCPC

Department of Anesthesiology, Pharmacology \& Therapeutics, The University of British Columbia, Medical Sciences Block C, 2176 Health Sciences Mall, Vancouver BC V6T 1Z3, Canada e-mail: stephan.schwarz@ubc.ca

Department of Anesthesia, St. Paul's Hospital/Providence Health Care, Providence Level 3, 1081 Burrard Street, Vancouver BC V6Z 1Y6, Canada the 2018 edition. $^{9}$ Regardless-or as a reflection-of the above, we suspect that few would doubt that these advances have greatly contributed to the level of patient care and safety ${ }^{10,11}$ that we have become accustomed to. That said, our sense is that a curiously persistent "urban myth" seems to prevail that we will never see a randomized comparison of general anesthesia with (vs without) the use of such a standard monitor as a continuous pulse oximeter-despite such compelling evidence to the contrary as the landmark study by Moller et al., reported in two seminal companion papers in 1993 (more on that below). ${ }^{3,12}$

In the current era of anesthesiology and perioperative medicine, we are witnessing the rapid evolution of another "thermometer" towards becoming a ubiquitous standard diagnostic modality in our specialty-point-of-care ultrasound (PoCUS). ("Rapid" may not be the most appropriate term as the concept of PoCUS has existed since the 1970s in Europe and Japan; in Canada, PoCUS started to take off after 2001 and subsequently became the focus of a dedicated society, the Canadian Point-of-Care Ultrasound Society [CPoCUS]). ${ }^{\text {A }}$ Among the myriad of PoCUS applications is gastric ultrasonography, ${ }^{13,14}$ which is of particular significance in anesthesiology and perioperative medicine as it speaks to one of the most feared anesthetic complications: pulmonary aspiration of gastric contents. ${ }^{15}$ In this edition of the Journal, Chang et al. ${ }^{16}$ report on the findings of a prospective observational cohort study in which patients scheduled for elective laparoscopic cholecystectomy underwent gastric ultrasound examinations prior to induction of anesthesia.

\footnotetext{
A Canadian Point of Care Ultrasound Society. Available from URL: https://www.cpocus.ca/about-ultrasound-society/history-ceus/ (accessed March 2020).
} 
The results are an eye-opener: the incidence of sonographic evidence of a full stomach among 138 Korean patients who had adhered to a standard fasting protocol (a minimum of two hours for clear fluid and $8 \mathrm{hr}$ for solid intake) was a whopping $13 \%$ ( $4.3 \%$ with an estimated $>1.5 \mathrm{~mL} \cdot \mathrm{kg}^{-1}$ of fluid, and a particularly troubling $8.7 \%$ with solid content).

That said, the results echo, at least qualitatively, previous reports by others. For example, Van de Putte $e t$ al. ${ }^{17}$ found a $6.2 \%$ incidence of a "full stomach" (used by Chang et al. as a reference/control incidence) in 538 fasted Belgian patients scheduled for a wide range of elective surgical procedures under general anesthesia; $4.5 \%$ had evidence of clear fluid $>$ $1.5 \mathrm{~mL} \cdot \mathrm{kg}^{-1}$ and $1.7 \%$ showed solid content. Another example is the recent report by Ohashi et al. on a mixed cohort of 222 Australian patients presenting for elective or expedited surgery: $49.5 \%$ had an empty stomach, $4.1 \%$ had a gastric residual volume $>100 \mathrm{~mL}$, and $2.7 \%>$ $1.5 \mathrm{~mL} \cdot \mathrm{kg}^{-1} .^{18}$ Nevertheless, while some hence may argue that the findings of Chang et al. are neither novel nor surprising, they are worrisome from a quantitative point of view. Several aspects amplify the disconcerting flavour of the report: elective laparoscopic cholecystectomy is a very common procedure; it appears difficult to imagine that the incidence in North American patients would be lower (to say the least) than in the Korean cohort surveyed by Chang et al.; and the suitability of supraglottic devices for airway management of these procedures has been proposed in the literature for quite some time now. ${ }^{19}$ Overall, the findings of Chang et al. certainly appear to support the notion that gastric emptying is delayed in patients with gallbladder disease, which may well have contributed to the comparatively high incidence of full stomach despite fasting. ${ }^{20-22}$

At the same time, the results of Chang et al. should be interpreted with caution as the study has a number of limitations. The design is an observational single cohort study. The study is devoid of a direct (or matched) control group. Allocation of patients to ultrasound examination was not random. Patients were not stratified according to (other) risk factors for delayed gastric emptying. Therefore, the results might have been influenced by biases and measurement errors. Examination of the initial ClinicalTrials.gov registration (NCT03259841) indicates a prespecified sample size of convenience of 240 in the context of an observational study design while the manuscript details a target sample size of 150 , calculated on the basis of null hypothesis testing (with a stated intent to identify a minimum important increase in the incidence of a full stomach of $6.2 \%$ in absolute terms from the assumed baseline of $6.2 \%$ reported by Van de Putte $e t$ al.). This raises concerns associated with post hoc protocol modification. Further, as indicated above, extrapolation of data from a Belgian cohort to Korean patients (and vice versa) requires caution. Finally, as pulmonary aspiration in elective surgical patients is a relatively rare event, ${ }^{23-25}$ neither the design nor the sample size permits valid inferences about this hard (and all-important) outcome. Collectively, the above gives the study and its results an exploratory, hypothesis-generating character.

Notwithstanding these considerations, from a clinical point of view, the findings by Chang et al. raise the question of what we ought to do if our friendly perioperative PoCUS colleague told us that our next elective patient has sonographic evidence of a full stomach despite fasting. The most striking aspect of Chang et al.'s report in that regard arguably is that is no case with a demonstrated full stomach was surgery delayed, postponed, or cancelled. Surgery proceeded as scheduled in all identified patients, with the sole modification being a rapid sequence induction (RSI). In contrast, in the study by Van de Putte et al. ${ }^{17}$ which Chang et al. used as a reference point, of the nine $(1.7 \%)$ patients found to have solid content, surgery was cancelled in one, delayed in two, and converted to a (loco)regional technique in three patients. In the remaining three patients, a decision was made to proceed with an RSI. As for the $23(4.5 \%)$ patients found to have clear fluid $>1.5 \mathrm{~mL} \cdot \mathrm{kg}^{-1}$, anesthetic management was changed in 16 patients: in ten patients, surgery was delayed until ultrasonography showed the stomach to be empty (after two to four hours), and in six patients an RSI was performed.

These differences raise a number of points. First, neither study-remarkably, some would argue-included a prespecified protocolized approach of what to do when a full stomach is identified. Second, as for proceeding with an RSI in elective patients with evidence of a full stomach, the question arises as to the supporting data for such an approach. In a review of randomized controlled trials in this Journal, Neilipovitz and Crosby found a notable absence of evidence for the efficacy of RSIs in decreasing the incidence of aspiration. ${ }^{26}$ A recent Cochrane Review arrived at similar conclusions for cricoid pressure. ${ }^{27}$ Third, many would likely argue that, if patient safety and wellbeing are the first priority, no meritorious argument supports elective laparoscopic surgery if there is evidence of a full stomach. Others, on the other hand, may contend that cancelling surgery is not a guarantee of overall safety either, since recurrence/development of acute cholecystitis or progression of disease before the next available surgical date are a distinct possibility - and that these risks may be higher, at least in terms of probability, than the risk of significant pulmonary aspiration. It might also be argued there is no guarantee that cancellation will result in a different finding on a different day.

Nevertheless, a useful feature of ultrasound is that it lends itself well to delay and serial re-scan-with or without the administration of a gastric motility agent- to 
confirm transition of a full stomach to an "empty" state. In addition to such a "delay and re-scan" approach as performed in ten patients in the above-referenced study by Van de Putte et al., ${ }^{17}$ a recent case report has described a similar strategy whereby ultrasound was used to assess the effect of prokinetic therapy with domperidone and erythromycin in an elective multimorbid patient who presented with a full stomach. ${ }^{28}$ Feasibility (and potential prokinetic drug adverse event) issues aside, even in the absence of robust research data, there appears to be little safety risk associated with delaying elective surgery until a full stomach has emptied compared with the potentially serious or catastrophic consequences-however rarepotentially associated with proceeding. Because the frequency of aspiration is low, the absence of documented instances in either study does not permit valid inferences about specific management strategies.

Chang et al. hence correctly observe in their discussion that further studies are required to corroborate the clinical implications of gastric ultrasound assessment for determining the aspiration risk in perioperative patient management. ${ }^{16}$ This statement echoes similar conclusions previously made by others. ${ }^{17,18,29}$ But back to the example of pulse oximetry: Moller et al.'s landmark randomized study in 20,802 patients, published in 1993 in two companion papers $^{3,12}$ along with a notable accompanying editorial, ${ }^{30}$ found that oximetry increased the detection of hypoxemia and reduced the incidence of myocardial ischemia, but did not decrease the overall rate of postoperative complications. Fast forward two decades, a Cochrane Review on the same topic arrived at similar conclusions. $^{31}$ We continue to use oximetry daily-and would not start a case without it. As for gastric ultrasound, does a degree of cognitive dissonance or a fixation error therefore exist when it comes to the conclusions drawn from the noted absence of studies on hard outcomes? Why should the burden of proof be that gastric ultrasound (and resultant management change) decreases morbidity and/or mortality, as opposed to that delaying cases until resolution of a full stomach status demonstrated by ultrasound is ineffective in minimizing the risk of aspiration?

In a recent prospective randomized and blinded study with healthy volunteers, Kruisselbrink et al. found that gastric ultrasound is highly sensitive and specific at detecting a "full stomach", and-arguably most importantly-has a negative predictive value of $100 \% .^{32}$ The alternative or "control" should also be carefully considered. While the pre-ultrasound era standard for many other PoCUS applications was "lowertech" diagnostic equipment such as a stethoscope, the equivalent for gastric ultrasound is the patient's fasting history. Is it rational to, on the one hand, cancel an elective case (as we have repeatedly done, and we suspect many others have also) if the patient drank a cup of cappuccino on the way to the hospital, but on the other hand carry on with an RSI in a fasted elective patient whose ultrasound exam shows a full stomach? We have our doubts. There is little doubt, however, that we all have been anesthetizing elective patients with full/partly full stomachs-mostly unknowingly, although sometimes it might have become horribly apparent upon laryngoscopy or extubation/ laryngeal mask removal, if not intraoperatively. There is also little doubt that gastric ultrasound can prevent many such events. Some might even argue that, in the PoCUS era, aspiration of solid stomach contents/particulate matter in elective patients should be added to the list of "never events" in anesthesia. ${ }^{33}$

Point-of-care ultrasound, taught in some form in all Canadian anesthesiology residency programs now, ${ }^{34}$ is here to stay. Like pulse oximetry and other standard monitors, PoCUS is non-invasive, safe, and quick. It accurately detects a "full stomach", and notably more so than pre-induction fasting, as impressively illustrated by Chang et al. in this edition of the Journal.

As for the question what to do after our friendly perioperative PoCUS colleague tells us that our next elective patient has sonographic evidence of a full stomach despite fasting, we believe we ought to practice with the same degree of caution as we would after obtaining a history of fasting protocol violation, which ordinarily would imply delay or cancellation-primum non nocere.

There is little doubt that further research is needed. However, is it still rational to consider taking a fasting history a mandatory part of routine pre-anesthetic assessment but not such an objective and highly accurate modality as gastric ultrasound $?^{18,35,36}$ In 2020 , the latter is far from new. ${ }^{14}$ It has previously been suggested that "the small number of unexpected 'full stomachs' does not justify an ultrasound examination in every patient presenting for elective surgery." 17 The report by Chang et al. in this edition of the Journal that $8.7 \%$ of fasted elective cholecystectomy patients had evidence of solid stomach content puts a different light on this. The article highlights an area of central importance to our specialty and underscores the mounting evidence that a history of fasting does not mean that the stomach is empty. As observed above, the path to new diagnostic tools and clinical standards takes time. We believe the time has come to move towards embracing gastric ultrasound as a routine part of future preoperative assessment-from our perspective, in the spirit of an enthusiastic rejection of the tenth Law of The House of God's cynicism. 


\section{Que faire quand}

l'échographie périopératoire

au chevet montre un

\section{estomac plein malgré un jeûne approprié?}

Pour citer le dixième Commandement de The House of God, le roman culte de 1978 sur la résidence en médecine : «Si vous ne prenez pas la température, vous ne trouverez pas de fièvre. ${ }^{1}$ Historiquement, en tant qu'anesthésiologistes, nous ne sommes pas les premiers concernés par le principe décidément cynique de ce commandement - soit de faire exprès d'éviter de chercher des problèmes. Bien au contraire : l'anesthésiologie a toujours été une spécialité qui, tout au long de son histoire, a vu l'arrivée et l'introduction d'une myriade de 'thermomètres' innovants, c'est-à-dire des outils de monitorage qui favorisent la détection en temps réel d'états physiopathologiques malencontreux, détection qui nous rend plus susceptibles de prendre une décision rapide. Les exemples les plus frappants sont peut-être la mesure de l'oxygénation du sang par des électrodes, puis plus tard par l'oxymétrie de pouls, ${ }^{2,3}$ la capnométrie, ${ }^{4}$ et le monitorage des agents anesthésiques. ${ }^{5}$

Tout cela est très bien, mais il faut du temps avant que ces outils diagnostiques deviennent des normes cliniques omniprésentes. Par exemple, l'édition révisée du Guide d'exercice de l'anesthésie de la Société canadienne des anesthésiologistes de 2012 recommandait que le monitorage par capnographie soit utilisé chez tous les patients pour lesquels une anesthésie générale ou des niveaux profonds de sédation étaient réalisés ${ }^{6}$ (cette recommandation a été étendue pour que la pratique soit envisagée pour tous les niveaux de sédation en 2019). ${ }^{7}$ En parlant de thermomètres, ${ }^{8}$ l'importance cruciale du maintien de la normothermie d'un patient en période périopératoire a été soulignée dans l'édition de 2018. ${ }^{9}$ Indépendamment - ou au vu - de ce qui précède, il est probable que tout le monde s'accorde sur le fait que ces avancées constituent une contribution majeure au niveau élevé de soins et de sécurité ${ }^{10,11}$ des patients auquel nous sommes aujourd'hui habitués. Cela dit, nous sommes d'avis qu'un « mythe urbain » étonnamment tenace semble prédominer, selon lequel il n'a aura jamais de comparaison randomisée de l'anesthésie générale avec (versus sans) utilisation d'un moniteur standard tel qu'un saturomètre en continu - malgré les données probantes extrêmement convaincantes à l'effet du contraire rapportées par Moller et coll. en 1993 (que nous aborderons plus loin)., 3,12
Dans notre ère actuelle d'anesthésiologie et de médecine périopératoire, nous assistons à l'évolution d'un autre «thermomètre » en passe de devenir rapidement une modalité diagnostique standard omniprésente de notre spécialité - l'échographie ciblée (PoCUS en anglais). («Rapidement» n'est peut-être pas le terme le plus adapté, étant donné que le concept d'échographie ciblée existe depuis les années 1970 en Europe et au Japon; au Canada, l'échographie ciblée a pris son envol après 2001 et est par la suite devenue le centre d'intérêt d'une société dédiée, la Société canadienne d'échographie ciblée [SCÉC]). ${ }^{\text {A }}$ Parmi les innombrables applications de l'échographie ciblée se trouve l'échographie gastrique, ${ }^{13,14}$ qui a une importance spéciale pour l'anesthésiologie et la médecine périopératoire étant donné qu'elle touche à l'une des complications anesthésiques les plus redoutées : l'aspiration pulmonaire du contenu gastrique. ${ }^{15}$ Dans ce numéro du Journal, Chang et coll. ${ }^{16}$ rapportent les résultats d'une étude de cohorte observationnelle prospective dans laquelle des patients devant subir une cholécystectomie par laparoscopie non urgente ont passé des examens échographiques gastriques avant l'induction de l'anesthésie. Les résultats sont saisissants: l'incidence de données probantes échographiques attestant d'un estomac plein parmi 138 patients coréens ayant respecté un protocole de jeûne standard (un minimum de deux heures pour les liquides clairs et huit heures pour les aliments solides) a atteint le chiffre énorme de $13 \%\left(4,3 \%\right.$ avec $>1,5 \mathrm{~mL} \cdot \mathrm{kg}^{-1}$ de liquide, et une proportion particulièrement troublante de $8,7 \%$ avec des aliments solides).

Ces résultats font toutefois écho, du moins d'un point de vue qualitatif, aux comptes rendus précédents réalisés par d'autres. Par exemple, Van de Putte et coll. ${ }^{17}$ ont observé une incidence de $6,2 \%$ d' « estomacs pleins » (utilisée par Chang et coll. comme incidence de référence/témoin) chez 538 patients belges à jeûne devant subir un vaste éventail d'interventions chirurgicales non urgentes sous anesthésie générale; chez $4,5 \%$ des patients, on a détecté la présence de $>1,5 \mathrm{~mL} \cdot \mathrm{kg}^{-1}$ de liquides clairs, et chez $1,7 \%$ des patients, la présence de contenu solide. Le récent compte-rendu de Ohashi et coll. sur une cohorte mixte de 222 patients australiens se présentant pour une chirurgie non urgente ou accélérée en est un autre exemple : 49,5\% avaient un estomac vide, $4,1 \%$ présentaient un volume résiduel gastrique $>100 \mathrm{~mL}$, et $2,7 \%$ avaient $>1,5$ $\mathrm{mL} \cdot \mathrm{kg}^{-1}{ }^{18}$ Toutefois, bien qu'on puisse arguer que les résultats de Chang et coll. ne soient ni nouveaux ni surprenants, ils demeurent cependant inquiétants d'un point de vue quantitatif. Divers aspects amplifient la saveur déconcertante de ce compte rendu : les cholécystectomies non urgentes par laparoscopie sont des interventions courantes; il semble difficile d'imaginer que l'incidence 
chez les patients nord-américains soit plus faible (c'est peu dire) que dans la cohorte coréenne de l'étude de Chang et coll.; et l'utilisation des dispositifs supraglottiques pour la prise en charge des voies aériennes de ces interventions a depuis longtemps été proposée dans la littérature. ${ }^{19}$ Globalement, les résultats de Chang et coll. semblent définitivement appuyer l'idée que la vidange gastrique est retardée chez les patients atteints de maladies vésiculaires, ce qui pourrait bien avoir contribué à l'incidence comparativement élevée d'estomacs pleins malgré le jeûne. ${ }^{20-22}$

Les résultats de Chang et coll. doivent tout de même être interprétés avec prudence, l'étude comportant plusieurs écueils : la conception de l'étude est celle d'une cohorte observationnelle unique; l'étude n'a pas de groupe témoin direct (ou apparié); l'attribution des patients au groupe d'examen échographique n'était pas aléatoire; et les patients n'ont pas été stratifiés selon (d'autres) facteurs de risque de retard de la vidange gastrique. Ainsi, les résultats peuvent avoir été influencés par des biais et des erreurs de mesure. L'inscription initiale auprès de ClinicalTrials.gov (NCT03259841) indique une taille d'échantillon de commodité pré-spécifiée de 240 patients dans le cadre d'une méthodologie d'étude observationnelle, alors que le manuscrit décrit une taille d'échantillon cible de 150 , calculée sur la base d'un test d'hypothèse nulle (avec une intention déclarée d'identifier une augmentation importante minimale de l'incidence d'estomac plein de 6,2 $\%$ en termes absolus à partir de la valeur de base de $6,2 \%$ rapportée par Van de Putte et coll.). Cela soulève des inquiétudes liées à la modification post-hoc du protocole. En outre, comme indiqué plus haut, il faut faire preuve de prudence lorsqu'on extrapole des données d'une cohorte belge à une cohorte de patients coréens (et vice versa). Enfin, étant donné que l'aspiration pulmonaire chez les patients de chirurgie non urgente est une complication relativement rare $^{23-25}$ ni la conception ni la taille d'échantillon ne permettent d'inférences valables concernant ce résultat clairement quantifiable (et ô combien important). Pris ensemble, tous les points énumérés ci-dessus donnent à l'étude et à ses résultats un caractère exploratoire et générateur d'hypothèses.

Indépendamment de ces réserves, d'un point de vue clinique, les observations de Chang et coll. soulèvent la question de savoir ce que nous devrions faire si un cher collègue venait nous voir après avoir réalisé une échographie ciblée périopératoire pour nous annoncer que notre prochain patient électif/non urgent montrait des signes échographiques d'estomac plein malgré son respect du jeûne. L'aspect le plus frappant du compte rendu de Chang et coll. à cet égard est qu'aucune chirurgie n'a été retardée, reportée ou annulée suite à un constat d'estomac plein. Les chirurgies ont eu lieu telles que prévues chez tous les patients identifiés, la seule modification résidant dans une induction en séquence rapide (ISR). En revanche, dans l'étude de Van de Putte et coll., ${ }^{17}$ utilisée comme point de référence par Chang et coll., sur les neuf $(1,7 \%)$ patients chez lesquels du contenu solide a été observé, une chirurgie a été annulée, deux ont été retardées, et trois ont été converties en technique (loco)régionale. Chez les trois autres patients, la décision de procéder en ISR a été prise. En ce qui a trait aux $23(4,5 \%)$ patients chez lesquels $>1,5$ $\mathrm{mL} \cdot \mathrm{kg}^{-1}$ de liquides clairs avaient été décelés, la prise en charge anesthésique a été modifiée pour 16 patients : dix chirurgies ont été retardées jusqu'à ce que l'échographie montre un estomac vide (après deux à quatre heures), et six ISR ont été réalisées.

Ces différences soulèvent plusieurs questions. Premièrement, aucune des deux études - ce qui pourrait être perçu comme remarquable - n'a inclus d'approche protocolaire pré-spécifiée concernant la marche à suivre en cas de détection d'un estomac plein. Deuxièmement, en ce qui touche à la réalisation d'une ISR chez les patients de chirurgie non urgente dont l'estomac était encore plein, la question de savoir quelles données appuient une telle approche se pose. Dans un compte rendu d'études randomisées contrôlées publié dans le Journal, Neilipovitz et Crosby ont remarqué l'absence notable de données probantes soutenant l'efficacité des ISR pour réduire l'incidence d'aspiration. ${ }^{26}$ Un compte rendu récent de la Cochrane Review est parvenu à des conclusions similaires en ce qui a trait à la pression cricoïdienne. ${ }^{27}$ Troisièmement, d'aucuns diraient probablement que, si la sécurité et le bien-être des patients sont véritablement la priorité première, aucun argument ne peut justifier une chirurgie non urgente par laparoscopie s'il y a preuve d'un estomac plein. En revanche, d'autres seraient d'avis que l'annulation de la chirurgie ne constitue pas non plus une garantie de sécurité globale, étant donné que la récurrence ou l'apparition d'une cholécystite aigüe ou la progression de la maladie avant la prochaine date chirurgicale disponible sont des possibilités bien réelles - et que ces risques pourraient être plus élevés, du moins en termes de probabilité, que le risque d'aspiration pulmonaire majeure. On pourrait tout aussi bien arguer qu'il n'y a aucune garantie que l'annulation entraîne un résultat différent un autre jour.

Toutefois, une caractéristique utile de l'échographie est qu'elle se prête bien aux délais et aux examens en série avec ou sans administration d'un agent de motilité gastrique - afin de confirmer la transition d'un estomac plein vers un état « vide». En plus d'une telle approche de « retarder et réitérer l'examen » telle qu'elle a été adoptée pour dix patients de l'étude susmentionnée de Van de Putte et coll., ${ }^{17}$ une présentation de cas récente a décrit une stratégie similaire dans laquelle l'échographie a été utilisée 
pour évaluer l'effet d'un traitement prokinétique à base de dompéridone et d'érythromycine chez un patient présentant de multiples morbidités devant subir une chirurgie non urgente et donc l'estomac était plein. ${ }^{28}$ Hormis les questions de faisabilité (et les effets secondaires négatifs potentiels des prokinétiques), même en l'absence de données de recherches robustes, il semble y avoir peu de risques d'innocuité liés à la décision de retarder une chirurgie non urgente jusqu'à ce qu'un estomac plein se vide par rapport aux conséquences potentiellement sérieuses voire catastrophiques - même si très rares associées à une chirurgie suivant son cours comme prévu. Parce que la fréquence d'aspiration est faible, l'absence d'instances documentées dans l'une et l'autre étude ne permet pas de tirer des conclusions valables concernant des stratégies de prise en charge spécifiques.

Chang et coll. ont donc correctement observé, dans leur discussion, que des études supplémentaires sont nécessaires pour corroborer les implications cliniques d'une évaluation échographique du contenu gastrique dans le but de déterminer le risque d'aspiration pour la prise en charge périopératoire des patients. ${ }^{16}$ Cette déclaration fait écho aux conclusions semblables précédemment tirées par d'autres. ${ }^{17,18,29}$ Mais revenons à l'exemple de l'oxymétrie de pouls : l'étude randomisée historique de Moller et coll. réalisée auprès de 20802 patients, publiée en 1993 dans deux articles conjoints ${ }^{3,12}$ en plus d'un éditorial complémentaire, ${ }^{30}$ est parvenue à déterminer que l'oxymétrie augmentait le dépistage de l'hypoxémie et réduisait l'incidence d'ischémie myocardique, mais que cette modalité ne réduisait pas le taux global de complications postopératoires. Vingt ans plus tard, une évaluation de la Cochrane Review sur le même sujet est parvenue à des conclusions similaires. ${ }^{31}$ Nous continuons d'utiliser l'oxymétrie quotidiennement et ne commencerions pas un cas sans y avoir accès. En ce qui a trait à l'échographie gastrique, existe-t-il alors un degré de dissonance cognitive ou une erreur de fixation lorsqu'il est question de tirer des conclusions malgré l'absence notée d'études sur les résultats clairement quantifiables? Pourquoi faudrait-il que la conclusion soit que l'échographie gastrique (et les modifications de prise en charge qui en résultent) réduit la morbidité et/ou la mortalité, plutôt que de conclure qu'il est inefficace de retarder les cas jusqu'à résolution de l'état d'estomac plein tel que démontré par l'échographie pour minimiser le risque d'aspiration?

Dans une récente étude randomisée prospective en aveugle, réalisée auprès de volontaires sains, Kruisselbrink et coll. ont observé que l'échographie gastrique était très sensible et spécifique pour dépister un « estomac plein », et - fait peut-être plus important encore - qu'elle a une valeur prédictive négative de $100 \%{ }^{32}$ L'alternative ou «témoin» devrait également être soigneusement examinée. Alors qu'à l'ère pré-échographique, la norme pour de nombreuses autres applications d'échographie ciblée consistait en équipements diagnostiques «moins technologiques » tels que le stéthoscope, la norme correspondant à l'échographie gastrique était: les antécédents de jeûne du patient. Dès lors est-il raisonnable, d'une part, d'annuler un cas non urgent (comme nous l'avons fait à maintes reprises, et nous ne sommes probablement pas les seuls) si notre patient a pris un cappuccino en chemin vers l'hôpital, mais d'autre part de réaliser une ISR chez un patient non urgent ayant jeûné mais dont l'examen échographique révèle un estomac plein? Probablement pas. Il ne fait que peu de doute, toutefois, que nous avons tous anesthésié des patients de chirurgie non urgente dont l'estomac était plein ou partiellement plein - la plupart du temps sans en être conscients, bien que, parfois, ces circonstances soient devenues affreusement apparentes lors d'une laryngoscopie ou de l'extubation/du retrait du masque laryngé, si ce n'est en période peropératoire. Il est tout aussi certain que l'échographie gastrique peut prévenir bon nombre de tels événements. Certains pourraient même dire que, à l'ère de l'échographie ciblée, une aspiration du contenu solide de l'estomac ou de matière particulaire chez des patients de chirurgie non urgente devrait être ajoutée à la liste des « événements ne devant jamais survenir » anesthésie. ${ }^{33}$

L'échographie ciblée, aujourd'hui enseignée d'une façon ou d'une autre dans tous les programmes canadiens de résidence en anesthésiologie, ${ }^{34}$ est là pour rester. Tout comme l'oxymétrie de pouls et d'autres moniteurs standard, l'échographie ciblée est une modalité non invasive, sécuritaire et rapide. Elle dépiste de façon précise un « estomac plein », bien plus que le jeûne préinduction, comme Chang et coll. l'ont illustré de façon frappante dans leur article publié dans ce numéro du Journal.

En ce qui touche à la question de savoir quoi faire après que notre cher collègue d'échographie ciblée périopératoire nous ait annoncé que l'examen échographique de notre prochain patient en électif montrait un estomac plein malgré le jeûne, nous pensons qu'il faut faire preuve d'autant de prudence que si nous avions obtenu des antécédents de violation du protocole de jeûne, ce qui, en règle générale, voudrait dire un report ou une annulation primum non nocere.

Il ne fait aucun doute que des recherches supplémentaires sont nécessaires. Toutefois, demeure-t-il raisonnable de tenir compte des antécédents de jeûne 
comme élément obligatoire de l'évaluation anesthésique pré-induction de routine, mais pas d'une modalité aussi objective et précise que l'échographie gastrique? ${ }^{18,35,36} \mathrm{En}$ 2020, la seconde modalité est loin d'être une nouveauté. Il a été suggéré par le passé que «le faible nombre 'd'estomacs pleins' imprévus ne justifie pas de réaliser un examen échographique chez chaque patient se présentant pour une chirurgie non urgente. ${ }^{17}$ Selon le compte rendu de Chang et coll. publié dans ce numéro du Journal, 8,7 \% des patients de cholécystectomie non urgente à jeun montraient des signes de contenu solide dans leur estomac : voilà qui apporte un éclairage différent à cette question. L'article met en lumière un domaine crucial de notre spécialité et souligne les données probantes croissantes selon lesquelles des antécédents de jeûne ne sont pas nécessairement synonymes d'un estomac vide. Comme observé ci-dessus, le chemin vers de nouveaux outils diagnostiques et de nouvelles normes cliniques est long et laborieux. Nous pensons que le temps est venu d'adopter l'échographie gastrique dans notre routine pour l'évaluation préopératoire future - selon nous, en respectant l'esprit d'un rejet enthousiaste du Dixième commandement cynique de The House of God.

Acknowledgements The authors gratefully acknowledge the Department of Anesthesia, St. Paul's Hospital/Providence Health Care (Vancouver, B.C.) for ongoing academic support. Dr. Stephan K. W. Schwarz holds the Dr. Jean Hugill Templeton Chair in Anesthesia, supported by the Dr. Jean Templeton Hugill Endowment for Anesthesia Memorial Fund.

Disclosures Dr. Stephan K.W. Schwarz is a member of the Editorial Board of the Canadian Journal of Anesthesia.

\section{Conflict of interest None.}

Funding statement None.

Editorial responsibility This submission was handled by Dr. Philip M. Jones, Associate Editor, Canadian Journal of Anesthesia.

Remerciements Les auteurs tiennent à mentionner leur reconnaissance envers le département d'anesthésie de l'Hôpital St Paul / Providence Health Care (Vancouver, C.-B.) pour la constance de son soutien académique. Le Dr Stephan K. W. Schwarz est titulaire de la Chaire en anesthésie Dr. Jean Templeton Hugill, financée par le Fonds commémoratif pour le financement en anesthésie Dr. Jean Templeton Hugill.

Déclarations Le Dr Stephan K.W. Schwarz est membre du Comité de rédaction du Journal canadien d'anesthésie.

\section{Conflits d'intérêt Aucun.}

\section{Déclaration de financement Aucune.}

Responsabilité éditoriale Cet article a été traité par Dr Philip M. Jones, rédacteur adjoint, Journal canadien d'anesthésie.

\section{References}

1. Shem S. The House of God. New York: Dell Publising; 1978.

2. Clark LC Jr, Wolf R, Granger D, Taylor Z. Continuous recording of blood oxygen tensions by polarography. J Appl Physiol 1953; 6: 189-93.

3. Moller JT, Johannessen NW, Espersen K, et al. Randomized evaluation of pulse oximetry in 20,802 patients: II. Perioperative events and postoperative complications. Anesthesiology 1993; 78: 445-53.

4. Jaffe $M B$. Infrared measurement of carbon dioxide in the human breath: "breathe-through" devices from Tyndall to the present day. Anesth Analg 2008; 107: 890-904.

5. Westhorpe RN, Ball C. Anaesthetic agent monitoring. Anaesth Intensive Care 2010; 38: 979. DOI: https://doi.org/10.1177/ $0310057 X 1003800602$.

6. Merchant $R$, Chartrand D, Dain $S$, et al. Guidelines to the practice of anesthesia revised edition 2012. Can J Anesth 2012; 59: 63-102. DOI: https://doi.org/10.1007/s12630-011-9609-0.

7. Dobson G, Chow L, Flexman A, et al. Guidelines to the practice of anesthesia - revised edition 2019. Can J Anesth 2019; 66: 75108. DOI: https://doi.org/10.1007/s12630-018-1248-2.

8. Ball C, Westhorpe RN. Temperature monitoring. Anaesth Intensive Care 2010; 38: 413. DOI: https://doi.org/10.1177/ $0310057 X 1003800301$.

9. Dobson G, Chong M, Chow L, et al. Guidelines to the practice of anesthesia - revised edition 2018. Can J Anesth 2018; 65: 76-104. DOI: https://doi.org/10.1007/s12630-017-0995-9.

10. Lienhart A, Auroy $Y$, Pequignot F, et al. Survey of anesthesiarelated mortality in France. Anesthesiology 2006; 105: 1087-97.

11. Bainbridge D, Martin J, Arango M, Cheng D, Evidence-based Peri-operative Clinical Outcomes Research (EPICOR) Group. Perioperative and anaesthetic-related mortality in developed and developing countries: a systematic review and meta-analysis. Lancet 2012; 380: 1075-81.

12. Moller JT, Pedersen T, Rasmussen LS, et al. Randomized evaluation of pulse oximetry in 20,802 patients: I. Design, demography, pulse oximetry failure rate, and overall complication rate. Anesthesiology 1993; 78: 436-44.

13. Van de Putte P, Perlas A. Ultrasound assessment of gastric content and volume. Br J Anaesth 2014; 113: 12-22.

14. Jacoby J, Smith G, Eberhardt M, Heller M. Bedside ultrasound to determine prandial status. Am J Emerg Med 2003; 21: 216-9.

15. Mendelson $C L$. The aspiration of stomach contents into the lungs during obstetric anesthesia. Am J Obstet Gynecol 1946; 52: 191205.

16. Chang JE, Kim H, Won D, et al. Ultrasound assessment of gastric content in fasted patients before elective laparoscopic cholecystectomy: a prospective observational single-cohort study. Can J Anesth 2020; DOI: https://doi.org/10.1007/s12630020-01668-7.

17. Van de Putte P, Vernieuwe L, Jerjir A, Verschueren L, Tacken M, Perlas A. When fasted is not empty: a retrospective cohort study of gastric content in fasted surgical patients. Br J Anaesth 2017; 118: 363-71.

18. Ohashi $Y$, Walker JC, Zhang F, et al. Preoperative gastric residual volumes in fasted patients measured by bedside ultrasound: a prospective observational study. Anaesth Intensive Care 2018; 46: 608-13.

19. Maltby JR, Beriault MT, Watson NC, Fick GH. Gastric distension and ventilation during laparoscopic cholecystectomy: LMAClassic vs. tracheal intubation. Can J Anesth 2000; 47: 622-6.

20. Ibrarullah M, Mittal BR, Agarwal DK, Das BK, Kaushik SP. Gastric emptying in patients with gallstone disease with or 
without dyspepsia: effect of cholecystectomy. Aust N Z J Surg 1994; 64: 247-50.

21. Berhane T, Hausken T, Olafsson S, Sondenaa $K$. Ultrasound examination shows disturbed proximal gastric function in symptomatic gallstone patients with dyspepsia and improvement after cholecystectomy-gallstones and gastric emptying. Ultraschall Med 2006; 27: 451-5.

22. Di Ciaula A, Molina-Molina E, Bonfrate L, Wang DQ, Dumitrascu DL, Portincasa P. Gastrointestinal defects in gallstone and cholecystectomized patients. Eur J Clin Invest 2019; 49: e13066. DOI: https://doi.org/10.1111/eci.13066.

23. Warner MA, Warner ME, Weber JG. Clinical significance of pulmonary aspiration during the perioperative period. Anesthesiology 1993; 78: 56-62.

24. Mellin-Olsen J, Fasting S, Gisvold SE. Routine preoperative gastric emptying is seldom indicated. A study of 85,594 anaesthetics with special focus on aspiration pneumonia. Acta Anaesthesiol Scand 1996; 40: 1184-8.

25. Cook TM, Woodall N, Frerk C; Fourth National Audit P. Major complications of airway management in the UK: results of the Fourth National Audit Project of the Royal College of Anaesthetists and the Difficult Airway Society. Part 1: anaesthesia. Br J Anaesth 2011; 106: 617-31.

26. Neilipovitz DT, Crosby ET. No evidence for decreased incidence of aspiration after rapid sequence induction. Can J Anesth 2007; 54: 748-64.

27. Algie CM, Mahar RK, Tan HB, Wilson G, Mahar PD, WasiakJ. Effectiveness and risks of cricoid pressure during rapid sequence induction for endotracheal intubation. Cochrane Database Syst Rev 2015; 11: CD011656. DOI: https://doi.org/10.1002/ 14651858.CD011656.pub2.

28. Sebrechts T, Perlas A, Abbas S, Van de Putte P. Serial gastric ultrasound to evaluate gastric emptying after prokinetic therapy with domperidone and erythromycin in a surgical patient with a full stomach: a case report. A A Pract 2018; 11: 106-8.

29. Perlas A, Arzola C, Van de Putte P. Point-of-care gastric ultrasound and aspiration risk assessment: a narrative review. Can J Anesth 2018; 65: 437-48.

30. Eichhorn JH. Pulse oximetry as a standard of practice in anesthesia. Anesthesiology 1993; 78: 423-6.

31. Pedersen T, Nicholson A, Hovhannisyan K, Moller AM, Smith $A F$, Lewis SR. Pulse oximetry for perioperative monitoring. Cochrane Database Syst Rev 2014; 3: CD002013. DOI: https:// doi.org/10.1002/14651858.CD002013.pub3.

32. Kruisselbrink R, Gharapetian A, Chaparro LE, et al. Diagnostic accuracy of point-of-care gastric ultrasound. Anesth Analg 2019; 128: 89-95.

33. Adyanthaya SS, Patil V. Never events: an anaesthetic perspective. Contin Educ Anaesth Crit Care Pain 2014; 14: 197-201. DOI: https://doi.org/10.1093/bjaceaccp/mkt055.

34. Mok D, Schwarz SK, Rondi K. Point-of-care ultrasonography in Canadian anesthesiology residency programs: a national survey of program directors. Can J Anesth 2017; 64: 1023-36.

35. Benhamou D. Ultrasound assessment of gastric contents in the perioperative period: why is this not part of our daily practice? $\mathrm{Br}$ J Anaesth 2015; 114: 545-8.

36. van de Putte P, van Hoonacker J, Perlas A. Gastric ultrasound to guide anesthetic management in elective surgical patients noncompliant with fasting instructions: a retrospective cohort study. Minerva Anestesiol 2018; 84: 787-95.

Publisher's Note Springer Nature remains neutral with regard to jurisdictional claims in published maps and institutional affiliations. 